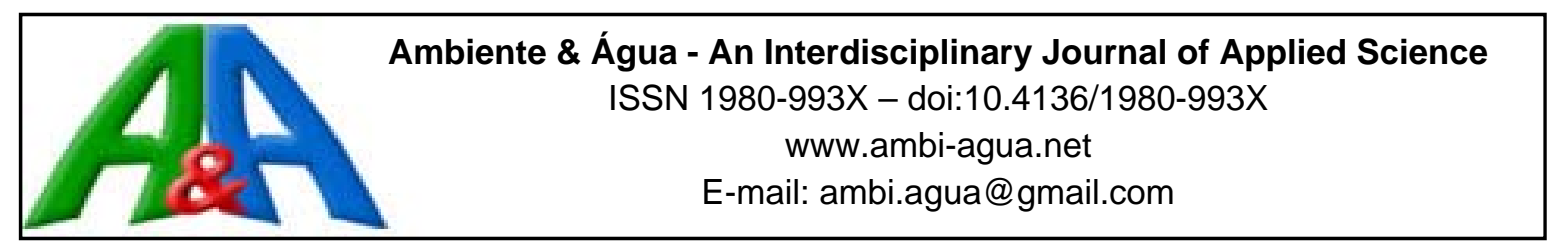

\title{
Modelado espacial de incendios: una herramienta predictiva para el Bosque La Primavera, Jalisco México
}

\author{
doi:10.4136/ambi-agua.1536
}

Received: 09 Oct. 2014; Accepted: 15 Dec. 2015

\author{
Jose Luis Ibarra-Montoya*; Francisco Martín Huerta-Martínez \\ Universidad de Guadalajara (UDG), Guadalajara, Jalisco, México \\ Centro Universitario de Ciencias Biológicas y Agropecuarias \\ Departamento de Ecología \\ *Autor correspondiente: e-mail: jibarra@ecologia.unam.mx, \\ fhuerta@cucba.udg.mx
}

\section{RESUMEN}

La interacción de diversos elementos de carácter socioeconómico, político y cultural, influidos por factores de paisaje y climáticos, son aspectos importantes en los regímenes de incendios. Sin duda la generación de modelos espaciales que integran esos elementos y factores, ayudan a predecir zonas potenciales de incendios con más exactitud. El Área de Protección de Flora y Fauna La Primavera (APFFLP) constituye el principal regulador climático de la zona metropolitana de Guadalajara, y presenta frecuentemente incendios forestales, por lo que representa un reto para la ciencia y la tecnología utilizarla como modelo para desarrollar metodologías que ayuden a predecir incendios forestales. El presente estudio se enfoca a la construcción de un modelo espacial que ayuda a determinar zonas potenciales de incendios en dicha área. El modelo integra variables meteorológicas, de paisaje, de combustibles, antropogénicas y/o de causalidad; y las ocurrencias históricas de los incendios comprendidos durante el periodo 1998-2012. Según el modelo, las variables que determinan las zonas con mayor potencial de incendios son: la pendiente (paisaje), la humedad relativa (meteorológica), el tipo de vegetación (causalidad) y el uso del suelo (antropogénicas). El modelo predice una zona extensa con alto potencial de incendiarse, ubicada en la parte central y noroeste del polígono del APFFLP; asimismo, existen zonas potenciales pequeñas y aisladas ubicadas en el oriente del polígono. La información generada en este estudio podría ayudar a la generación de mapas de riesgo locales y con ello, optimizar las acciones de manejo del fuego y restauración del Bosque La Primavera.

Palabras clave: área natural protegida, heterogeneidad ambiental, landscape, mapas de riesgo, MAXENT.

\section{Modelagem espacial de incêndios: uma ferramenta preditiva para o Bosque La Primavera, Jalisco México}

\section{RESUMO}

A interação de vários elementos de natureza socioeconômica, política e cultural, influenciados pela paisagem e fatores climáticos, são aspectos importantes do regime de fogo. Definitivamente, modelos espaciais que integram esses elementos ajudam a prever áreas 
potenciais de incêndio de forma mais precisa. A Área de Proteção da Vida Selvagem La Primavera (APFFLP) é o principal regulador do clima da região metropolitana de Guadalajara e, frequentemente, apresenta incêndios florestais, o que representa um desafio para a ciência e tecnologia o desenvolvimento de metodologias que possam ajudar prever estes incêndios. $\mathrm{O}$ presente estudo envolve a construção de um modelo espacial que ajuda a identificar áreas potenciais de incêndio na área. O modelo integra variáveis meteorológicas, paisagem, combustíveis, antropogênicas e/ou naturais; assim como, ocorrências históricas de incêndios durante o período 1998-2012. De acordo com o modelo, as variáveis que determinam as áreas de maior potencial de incêndios são: a declividade (paisagem), umidade relativa (tempo), tipo de vegetação (natural) e uso da terra (antropogênica). O modelo prediz uma grande área, com um elevado potencial de incêndio, localizado no polígono APFFLP central e noroeste; além disso, há pequenas zonas potenciais, isoladas ao leste do polígono. As informações geradas neste estudo podem dar suporte à geração de mapas de risco local e, assim, otimizar as ações de manejo do fogo e restauração do Bosque La Primavera.

Palavras-chave: área protegida, heterogeneidade ambiental, cenário, mapas de risco, MAXENT.

\title{
Spatial modeling of fires: a predictive tool for La Primavera Forest, Jalisco Mexico
}

\begin{abstract}
The interaction of various elements of socioeconomic, political and cultural nature, influenced by landscape and climatic factors, are important aspects of fire regimes. Space models that integrate these elements and factors help to more accurately predict potential fire areas. The Protected Area Wildlife La Primavera (APFFLP) is the main regulator of the climate of the Guadalajara metropolitan area, and forest fires frequently occur there. These represent a challenge for science and technology to develop methodologies that help predict forest fires. This study involves the construction of a spatial model that helps identify potential areas of fire in that area. The model integrates meteorological variables, landscape, fuels, anthropogenic and / or causality, and historical occurrences of fires during the period 1998-2012. According to the model, the variables that determine the areas of greatest fire potential are: slope (landscape), relative humidity (weather), vegetation type (causality) and land use (anthropogenic). The model predicts a large area with high potential for fire, located in the central and northwest APFFLP polygon; also, there are small, isolated potential zones in the eastern part of the polygon. The information developed by this study could support the generation of local risk maps, thereby optimizing the actions of fire management and restoration of the La Primavera forest.
\end{abstract}

Keywords: environmental heterogeneity, landscape, protected area, risk maps, MAXENT.

\section{INTRODUCCIÓN}

Los incendios forestales son el resultado de la interacción de diversos elementos de carácter socioeconómico, político y cultural que, influidos por factores de paisaje y climáticos, determinan la magnitud e intensidad de su comportamiento (Sánchez, 1989). Es por ello que predecir las ocurrencias de incendios forestales y entender sus factores asociados es un reto para la ciencia y es un proceso clave para diseñar estrategias de manejo del fuego (Gollberg et al., 2001). Diversos estudios se han enfocado a estudiar la interacción entre los factores físicos y climáticos del sitio (Flase y Ceccato, 1996; Sepúlveda et al., 2001; Giglio et 
al., 2003; Dryry y Veblen, 2008), en la mayoría de éstos se menciona que los incendios se correlacionan con las variaciones climáticas extremas y en algunos casos se ha explicado su ocurrencia desde una perspectiva geoespacial (Ávila-Flores y Pompa-Garcia, 2010). También se han desarrollado modelos que consideran las variables meteorológicas y de combustible para explicar el comportamiento de los incendios (Stolyarchuk, 1979; Mathur et al., 1984; Marcozzi et al., 1994; Mandallaz y Ye, 1997; Wybo et al., 1995; Gutiérrez et al., 1997; Gouma y Chronopoulou-Sereli, 1998; Leathwick y Briggs, 2001).

A nivel mundial existen sistemas que ayudan a identificar las áreas con peligro de incendio, destacan el Canadian Forest Fire Danger Index (Lee et al., 2002); Forest Fire Danger Index desarrollado en Australia (CSIRO, 2000) y el National Fire Danger Rating System, desarrollado por el Servicio Forestal de Estados Unidos de América (Deeming et al., 1978). En México no se cuenta con un sistema de identificación de áreas potenciales con peligro de incendio; es competencia del Centro Nacional de Control de Incendios Forestales (CENCIF) concentrar, procesar y distribuir la información de indicadores de riesgo y de los incendios forestales detectados en el territorio nacional a través de la detección satelital de puntos de calor (CONAFOR, 2014b; CONABIO, 2014).

Los modelos sobre incendios desarrollados para México han sido escasos y aislados y consideran solo la carga de combustibles forestales y algunas variables meteorológicas (Magaña, 1985; Zapata, 1991; Santillán, 1993). Por ejemplo, destacan los trabajos de Sepúlveda et al. (1999), quienes desarrollaron un modelo de combustibles en Baja California utilizando variables meteorológicas; Muñoz et al. (2005) generaron un modelo de comportamiento de incendios para la Sierra Madre Oriental y Pérez-Verdín et al. (2013) quienes desarrollaron un análisis espacio-temporal de la ocurrencia de incendios forestales en Durango, México; utilizando patrones de distribución espacial y análisis de estacionalidad y auto correlación de variables que afectan la magnitud de los mismos. Aún con estos esfuerzos, los modelos generados no utilizan todos los componentes y variables que interactúan para predecir la ocurrencia detallada de un incendio en una determinada zona; por lo que es necesario desarrollar modelos espaciales que integren variables meteorológicas, de combustibles y de paisaje con los factores de carácter socioeconómico, político y cultural y generar escenarios futuros de áreas potenciales de incendios, y puedan servir de apoyo a las autoridades correspondientes de combatir los incendios y en la toma de decisiones sobre la conservación, restauración y gestión ambiental.

El Área de Protección de Flora y Fauna Bosque La Primavera, (APFFLP), ubicada al oeste de la ciudad de Guadalajara, Jalisco, México, es el principal regulador del clima de la zona conurbada de Guadalajara, provee bienes y servicios a la población y alberga una gran diversidad de especies que mantienen una interacción con el bosque de encino y pino, y dependen casi exclusivamente de la existencia de este bosque y sus corredores ecológicos (Mexico, 2000). Desde el periodo comprendido entre los años 1910-1960, el Bosque La Primavera ha sido blanco del aprovechamiento intensivo de los recursos forestales maderables y no maderables, y se llevan a cabo actividades de agricultura, de uso público y especialmente la generación de incendios controlados durante la zafra en el cultivo de la caña de azúcar. Dadas estas actividades, el área presenta una alta frecuencia de incendios forestales que han llegado a afectar más de 11 mil hectáreas en un año (Mexico, 2012).

El objetivo del presente trabajo fue desarrollar un modelo espacial predictivo de áreas potenciales de incendios para el Área de Protección de Flora y Fauna Bosque La Primavera, México, tomando como base los datos históricos de la ocurrencia de incendios en el APFFLP durante el periodo 1998-2012 (Huerta-Martínez e Ibarra-Montoya, 2014) así como las variables de combustibles, meteorológicas, de paisaje y antropogénicas y/o de causalidad. 


\section{MATERIALES Y MÉTODOS}

\subsection{Sitio de estudio}

El APFFLP está ubicada en la región central del estado de Jalisco, entre las coordenadas extremas $103^{\circ} 28^{\prime}$ a $103^{\circ} 42^{\prime}$ de longitud oeste, y $20^{\circ} 32^{\prime}$ a $20^{\circ} 44^{\prime}$ de latitud norte. Está situada al centro de un conjunto de cinco valles (Tala, Tesistán, Toluquilla, Atemajac y San Isidro Mazatepec) pertenecientes a los municipios de Zapopan, Tala, y Tlajomulco de Zúñiga. La Primavera es el bosque más cercano a la ciudad de Guadalajara, localizándose al poniente de la misma, y está circundado por las siguientes carreteras: Guadalajara-Nogales al norte; Tala-Ahuisculco al oeste; Tlajomulco de Zúñiga-San Isidro Mazatepec al sur; y Guadalajara-Barra de Navidad y Periférico poniente de la Ciudad de Guadalajara desde la Avenida López Mateos hasta la Av. Vallarta, al este (Figura 1).

El APFFLP es un Área Natural Protegida (ANP) que tiene alrededor de 22 puntos de acceso mediante caminos de terracería y brechas, tres de los cuales se utilizan principalmente con fines recreativos: uno por la Av. Mariano Otero, en el lado oriente de esta zona, y dos en el lado norte, por el ejido La Primavera y Puente Las Tortugas.

Según la clasificación climática de Köppen, modificada por García (1973) para la República Mexicana, el clima predominante es templado subhúmedo $\mathrm{S}\left(\mathrm{w}_{1}\right)(\mathrm{w})$ y semicálido subhúmedo $(\mathrm{A}) \mathrm{C}\left(\mathrm{w}_{1}\right)(\mathrm{w})$. La temperatura media anual es de $20.6{ }^{\circ} \mathrm{C}$, siendo el mes más frío enero y el más cálido junio. El promedio anual de humedad es de 63\%. Los vientos dominantes provienen del suroeste, con una velocidad máxima $53 \mathrm{~km} \mathrm{~h}^{-1}$.

El APFFLP se asienta sobre la Sierra La Primavera, la cual se ubica en la provincia fisiográfica del Eje Neovolcánico, subprovincia Guadalajara, que se caracteriza por manifestaciones recientes de vulcanismo explosivo. Está considerada como la manifestación riolítica más reciente y compleja de toda la provincia del Eje Neovolcánico. Por su variación en cuanto a manifestaciones ígneas ácidas es uno de los relieves volcánicos con más diversidad de México, en el que se combinan domos anulares, mesetas, cerros y montañas que siguen las líneas de fractura de la caldera volcánica, y colinas irregulares modeladas por erosión. Si bien estas formas tienen un origen volcánico común, en la actualidad presentan influencia de las fuerzas denudatorias fluviales y tectónicas.

De acuerdo a la clasificación FAO/UNESCO, las unidades de suelo que constituyen el área comprendida en el APFFLP están representados por regosol y litosol. El regosol conforma el 92\% del ANP, el cual es derivado del intemperismo de la toba, pómez y riolita. En el restante $8 \%$ de la superficie en cuestión, se encuentran distribuidos suelos de tipo litosol, resultantes de procesos erosivos. Ambos suelos se caracterizan por presentar una profundidad efectiva máxima de acuerdo a lo siguiente: el $44 \%$ de la superficie con suelo presenta un espesor de 10 a $30 \mathrm{~cm}$; el $9 \%$ de 30 a $60 \mathrm{~cm}$ y el $47 \%$ restante es mayor de $60 \mathrm{~cm}$.

El $80 \%$ del suelo involucrado en el ANP presenta valores de contenido de materia orgánica menores al $2 \%$, lo que indica que no existe el suelo típico de bosque. La vegetación presente esta caracterizada principalmente por Bosque de encino, bosque de encino-pino, bosque de pino y bosque tropical caducifolio.

\subsection{Modelo conceptual}

Para la conformación del modelo espacial, fue necesario construir un modelo conceptual basado inicialmente en el publicado por Pompa (2012). Dicho modelo incluye cuatro componentes espaciales: 1) complejo de variables de combustibles, construido a partir de la existencia real de las especies dominantes (número); el $\mathrm{pH}$ del suelo; la profundidad de la hojarasca $(\mathrm{cm})$ y la concentración de carbono medida como la biomasa acumulada $\left(\mathrm{g} / \mathrm{cm}^{2}\right)$, con valores asignados en cada sitio de muestreo; 2) componente meteorológico, definido por la temperatura ambiental $\left({ }^{\circ} \mathrm{C}\right)$ y la humedad relativa $\left.(\%) ; 3\right)$ componente de paisaje, 
constituido por la pendiente (\%), la altitud (msnm), la exposición (azimut) y la fisiografía (raster); y 4) componente antropogénico y/o de causalidad, integrado por las variables de uso de suelo (raster), tipo de suelo (raster), tipo de vegetación (raster) y perturbación (\%). Su integración esquemática se muestra en la Figura 2.

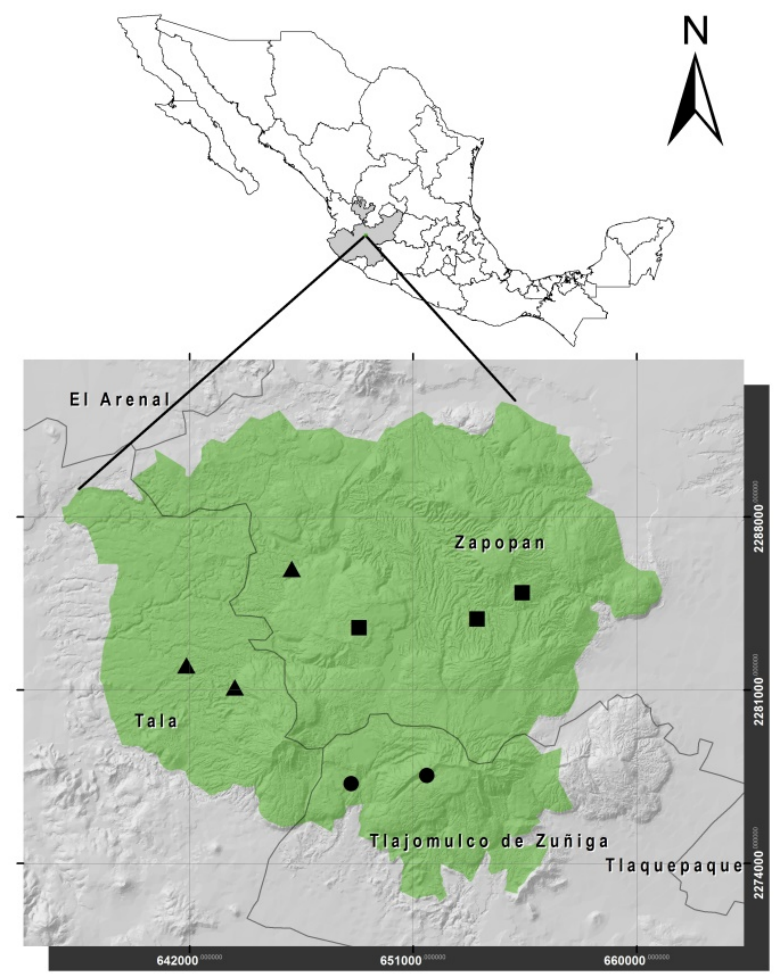

Figura 1. Mapa de localización geográfica del APFFLP, y ubicación de las estaciones de muestreo.

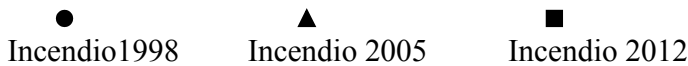

Modelo conceptual de la ocurrencia futura de incendios

- Especies dominantes

- $\mathrm{pH}$ del suelo

- Prof. de hojarasca

- Biomasa

Incendio1998

Incendio 2005

Incendio 2012

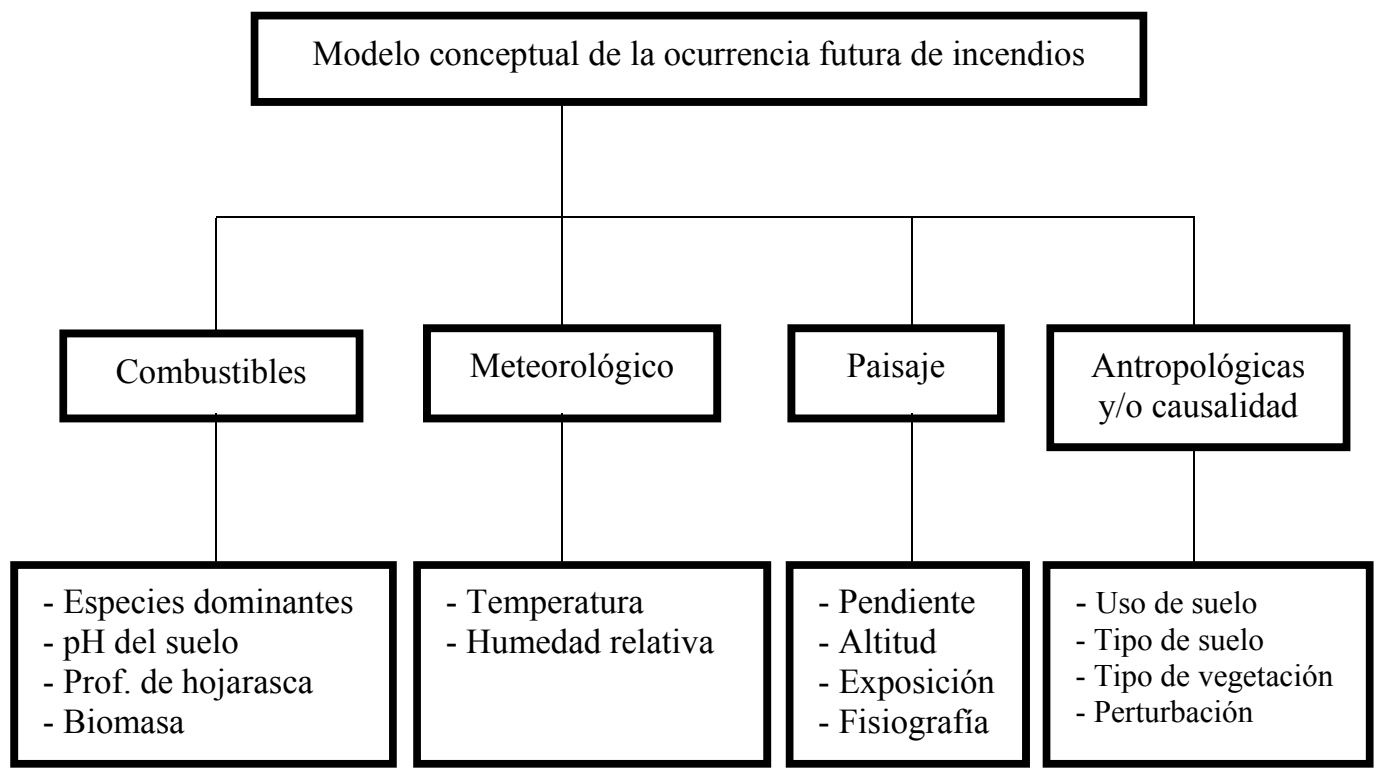

Figura 2. Esquema de la generación del modelo conceptual de ocurrencia futura de incendios forestales: Componentes y variables (Modificado de Pompa, 2012). 


\subsection{Selección de los puntos de muestreo}

Se eligieron ocho estaciones de muestreo ubicadas dentro del polígono del APFFLP; los sitios corresponden a las áreas quemadas por los incendios de los años: 1998, 2005 y

2012 (Figura 1, Tabla 1). Los sitios de muestreo fueron georreferenciados con un geoposicionador satelital (GPS map, $178 \mathrm{C}$ Sounder, marca GARMIN).

En cada una de las estaciones de muestreo se registraron catorce variables correspondientes al esquema del modelo conceptual de ocurrencia de incendios propuesto en este estudio (Figura 2).

Para el caso de la biomasa acumulada (combustibles forestales), la metodología consistió en la ubicación de una línea de $20 \mathrm{~m}$ de longitud cuya dirección fue definida aleatoriamente, para cada sitio de muestreo. Para el caso de la cama de combustibles forestales, se registró su profundidad a los 5, 10, 15, y $20 \mathrm{~m}$ de longitud de la línea. Además de colectó la hojarasca contenida en $1 \mathrm{~m}^{2}$ compuesto por cuatro cuadrantes de $0.25 \mathrm{~m}^{2}$ distribuidos a lo largo de la línea. Cada cuadrante fue pesado in situ y se tomó una muestra de $300 \mathrm{~g}$ para su posterior secado en el laboratorio. El peso seco de estas muestras fue relacionado con el peso del material colectado in situ y de esta forma se obtuvo la cantidad de este tipo de combustible por unidad de superficie.

Para determinar la altitud se empleó un modelo digital del terreno (MDT) con una resolución espacial de $30 \mathrm{~m}$, generado a partir de las curvas de nivel de INEGI; escala 1:50,000 (INEGI, 2014b).

Debido a la dificultad de representar espacialmente los agentes causales de incendios, y en virtud de la importancia de su integración en el modelo, se realizó el análisis de los rasgos geográficos asociados con actividades humanas. Chuvieco et al. (2000), García et al. (1999) y Almeida (1994), señalan que el desarrollo de un modelo de predicción de incendios siempre presenta dificultades debido a la incertidumbre asociada al comportamiento humano en relación con el fuego. En este caso los rasgos evaluados fueron el tipo de vegetación, uso de suelo, tipo de suelo y la perturbación.

La selección de las estaciones de muestreo, se basaron en encontrar los lugares adecuados para generar un mapa de interpolación por cada variable analizada. Por lo tanto las estaciones fueron seleccionadas según los criterios: i) ubicación dentro del área quemada para los años 1998, 2005 y 2012; ii) hacer una réplica en cada uno de los años; iii) incluir sitios con tipo de vegetación contrastante para análisis de la biomasa proveniente de diferentes especies de árboles.

\subsection{Identificación de las especies presentes en cada estación de muestreo}

Se identificaron las especies de árboles presentes en cada sitio, para ello se colectó material foliar de los individuos y se llevó a cabo la identificación utilizando las claves taxonómicas para encinos y pinos (Nixon y Muller, 1993; Vázquez et al., 2004; Manos et al., 1999); en aquellos organismos en donde se tuviera alguna duda se revisaron con el especialista. Se determinó que solo se analizaría la biomasa proveniente de los árboles (pinos y encinos) para dejar de lado la posible variación en la biomasa producida por las plantas anuales.

\subsection{Generación de coberturas de los parámetros}

Utilizando los datos obtenidos en campo de las diferentes variables, se construyeron las coberturas utilizando el polígono que delimita al APFFLP (Huerta-Martínez e IbarraMontoya, 2014). Estas coberturas, mapas tipo "raster" se generaron utilizando la técnica IDW de interpolación (Distancia Inversa Ponderada) (Delaney, 1999), en el Sistema de Información Geográfica ArcMap 9.2 ${ }^{\circledR}$ (GIS, ESRI, 1999-2001) enmascarando el análisis solo para el polígono del APFFLP. Para las variables de uso de suelo, tipo de vegetación, tipo de 
suelo y fisiografía, su utilizaron los shapes de INEGI (INEGI, 2014a), y el modelo digital de terreno (MDT). La resolución definitiva (tamaño de pixel) de todas las coberturas fue de $1 \mathrm{~m}^{2}$.

\subsection{Modelo espacial}

Se utilizó el enfoque de Máxima Entropía (MAXENT) para modelar las predicciones de incendios, para lo cual fue utilizada la versión de escritorio 3.3.0. de noviembre 2011 (Phillips et al., 2006). En general, este algoritmo detecta las relaciones no aleatorias entre dos conjuntos de datos; a) los registros georreferenciados de la presencia de los incendios, y b) un conjunto de coberturas tipo "raster", de datos digitales que representan a las variables de combustible, meteorológicas, de paisaje, antropológicas y de causalidad pertinentes para determinar las zonas potenciales de incendios (Phillips et al., 2006).

Una explicación detalla de los aspectos técnicos de MAXENT se puede revisar en Phillips et al. (2006), sin embargo el procedimiento general aplicado para construir los modelos espaciales en el APFFLP es el siguiente:

Utilizando las coberturas de los parámetros y los datos históricos (1998-2012) de ocurrencia de los incendios se llevó acabo el modelaje. El 75\% de los registros de presencia fueron utilizados como puntos de entrenamiento y el $25 \%$ como puntos de validación. Se utilizó un umbral de convergencia de $10^{-5}$ con 1000 iteraciones como límite superior para cada corrida. Para estimar la capacidad de predicción de cada modelo, se analizó el área bajo la curva (AUC), salida grafica donde se observa la capacidad de discriminación de una ausencia (especificidad) (Phillips y Dudik, 2008). El formato de salida analizado es el acumulativo (rango de 0 a 100, el cual indica una idoneidad de incendio potencial relativa de cada pixel mas no una probabilidad de ocurrencia del incendio (salida logística). Se uso la prueba de Jackknife (Sokal y Rohlf, 1995) para calcular la contribución relativa de cada variable al modelo, los resultados de esta evaluación se expresan con una medida conocida como "ganancia". Esta información es de suma importancia para evidenciar las características que deberían prescribir un incendio y que probablemente determinen el área potencial a incendiarse. Ambos análisis, el AUC y la prueba de Jacknife están implementados en MAXENT.

Debido a que MAXENT produce resultados diferentes de una corrida a la siguiente, utilizando la misma entrada de datos, se desarrollaron 100 modelos de predicción independientes para cada ocurrencia de incendio, y se eligieron un subconjunto de los mejores 10 modelos basado en dos criterios: i) fue seleccionado un primer conjunto de 20 modelos con menos del 10\% de error por omisión y, ii) de ellos, se seleccionaron los 10 modelos más cercanos a la media en las zonas donde han ocurrido los incendios. Estos 10 modelos fueron examinados en un Sistema de Información Geográfica (SIG), y se generó un mapa de consenso con los valores de pixel de 0 a 10, donde el 0 representa las áreas en las que todos los modelos predicen la ausencia de probabilidad de incendio y el 10 representa las zonas donde todos los modelos coinciden en la predicción de las zonas potenciales de incendios. Para disminuir la sobre predicción que pudiera presentar el modelo se decidió hacer un mapa utilizando las probabilidades entre $0.8,0.9$ y 1 .

\section{RESULTADOS Y DISCUSIÓN}

En el modelo espacial, la capacidad de predicción para los datos de prueba generó un AUC de 0.834 , lo que indica que la robustez del modelo para clasificar las presencias de los incendios fue buena (Parolo et al., 2008). Así mismo, las variables para todos los sitios que tuvieron el mayor poder de predicción cuando fueron analizadas con Jacknife de manera independiente fueron: la pendiente $(\mathrm{AUC}=0.91)$, la humedad relativa $(\mathrm{AUC}=0.82)$, el tipo de vegetación $(\mathrm{AUC}=0.83)$ y el uso de suelo $(\mathrm{AUC}=0.79)$, además, fisiografía resultó con poder 
de predicción en Rio Caliente, así como temperatura y pH en Cerritos Colorados (Tabla 2).

Tabla 1. Año de incendio y los sitios de muestreo. Se muestra también el uso de suelo y tipo de vegetación, así como las especies identificadas por cada localidad.

\begin{tabular}{|c|c|c|c|c|}
\hline Año & Localidad & U. suelo & Vegetación & Esp. Dominante \\
\hline \multirow{6}{*}{1998} & \multirow{4}{*}{1 Planillas } & \multirow{4}{*}{ Forestal } & \multirow{4}{*}{ Encino-Pino } & Quercus resinosa \\
\hline & & & & Quercus viminea \\
\hline & & & & Pinus oocarpa \\
\hline & & & & Q. coccolobifolia \\
\hline & \multirow{2}{*}{2 Poleo } & \multirow{2}{*}{ Forestal } & \multirow{2}{*}{ Pino-Encino } & Quercus resinosa \\
\hline & & & & Pinus oocarpa \\
\hline \multirow{7}{*}{2005} & \multirow{3}{*}{3 Cerro San Miguel } & \multirow{3}{*}{ Forestal } & \multirow{3}{*}{ Encino } & Quercus resinosa \\
\hline & & & & Quercus viminea \\
\hline & & & & Pinus oocarpa \\
\hline & \multirow{2}{*}{4 Cerro San Miguel 1} & \multirow{2}{*}{$\begin{array}{l}\text { Forestal/ } \\
\text { Pastoreo }\end{array}$} & \multirow{2}{*}{ Pino-Encino } & Quercus resinosa \\
\hline & & & & Pinus oocarpa \\
\hline & \multirow{2}{*}{5 Río Caliente } & \multirow{2}{*}{ Forestal } & \multirow{2}{*}{ Pino-Encino } & Quercus resinosa \\
\hline & & & & Pinus oocarpa \\
\hline \multirow{8}{*}{2012} & \multirow{2}{*}{6 Ejido López Mateos } & \multirow{2}{*}{ Forestal } & \multirow{2}{*}{ Pino-Encino } & Quercus resinosa \\
\hline & & & & Pinus oocarpa \\
\hline & \multirow{3}{*}{7 Cerritos Colorados } & \multirow{3}{*}{$\begin{array}{l}\text { Forestal/ } \\
\text { Zona } \\
\text { Geotermal }\end{array}$} & \multirow{3}{*}{ Pino-Encino } & Quercus resinosa \\
\hline & & & & Quercus viminea \\
\hline & & & & Pinus oocarpa \\
\hline & \multirow{3}{*}{8 Crucero Pedernal } & \multirow{3}{*}{ Forestal } & \multirow{3}{*}{ Pino-Encino } & Quercus castanea \\
\hline & & & & Quercus resinosa \\
\hline & & & & Pinus oocarpa \\
\hline
\end{tabular}

Tabla 2. Valores de AUC para catorce variables analizadas en los ocho sitios muestreados, según el modelo espacial. Las negritas significan un AUC $\geq 0.8$.

\begin{tabular}{lcccccccc}
\hline Variable & \multicolumn{10}{c}{ Localidad } \\
\hline & 1 & 2 & 3 & 4 & 5 & 6 & 7 & 8 \\
Tipo de Suelo & 0.64 & 0.68 & 0.34 & 0.48 & 0.49 & 0.42 & 0.36 & 0.45 \\
Humedad relativa & $\mathbf{0 . 8 4}$ & $\mathbf{0 . 8 0}$ & $\mathbf{0 . 8 5}$ & $\mathbf{0 . 8 3}$ & $\mathbf{0 . 8 9}$ & $\mathbf{0 . 8 6}$ & 0.69 & $\mathbf{0 . 8 9}$ \\
pH & 0.67 & 0.78 & 0.70 & 0.67 & 0.78 & 0.69 & $\mathbf{0 . 8 8}$ & 0.72 \\
Prof. Hojarasca & 0.38 & 0.47 & 0.49 & 0.40 & 0.67 & 0.74 & 0.47 & 0.47 \\
Biomasa & 0.45 & 0.56 & 0.67 & 0.58 & 0.49 & 0.55 & 0.62 & 0.61 \\
Temperatura & 0.63 & 0.76 & 0.65 & 0.37 & 0.71 & 0.79 & $\mathbf{0 . 8 3}$ & 0.73 \\
Fisiografía & 0.57 & 0.58 & $\mathbf{0 . 8 0}$ & 0.61 & $\mathbf{0 . 8 8}$ & 0.60 & 0.79 & 0.68 \\
Pendiente & $\mathbf{0 . 8 3}$ & $\mathbf{0 . 8 4}$ & $\mathbf{0 . 8 9}$ & $\mathbf{0 . 8 2}$ & $\mathbf{0 . 8 3}$ & $\mathbf{0 . 8 0}$ & $\mathbf{0 . 8 5}$ & $\mathbf{0 . 9 3}$ \\
Exposición & 0.67 & 0.57 & 0.68 & 0.69 & 0.51 & 0.55 & 0.75 & 0.78 \\
Perturbación & 0.70 & 0.79 & 0.62 & 0.69 & 0.79 & 0.61 & 0.61 & 0.68 \\
Uso de Suelo & $\mathbf{0 . 8 6}$ & $\mathbf{0 . 8 4}$ & $\mathbf{0 . 8 2}$ & $\mathbf{0 . 8 9}$ & $\mathbf{0 . 8 8}$ & $\mathbf{0 . 8 6}$ & $\mathbf{0 . 8 2}$ & $\mathbf{0 . 8 1}$ \\
Tipo de Vegetación & 0.78 & $\mathbf{0 . 8 9}$ & $\mathbf{0 . 8 4}$ & $\mathbf{0 . 8 9}$ & $\mathbf{0 . 8 7}$ & $\mathbf{0 . 8 8}$ & $\mathbf{0 . 8 3}$ & $\mathbf{0 . 8 9}$ \\
Altitud & 0.64 & 0.68 & 0.62 & 0.59 & 0.67 & 0.71 & 0.64 & 0.66 \\
Esp. Dominantes & 0.68 & 0.62 & 0.47 & 0.70 & 0.73 & 0.62 & 0.56 & 0.73 \\
\hline
\end{tabular}


Las predicciones del modelo espacial desarrollado en este trabajo muestran una zona continua con alto potencial de incendio ubicada en la parte central y noreste del polígono del APFFLP de aproximadamente 4,937 Ha (Figura 3), correspondiente al Ejido López Mateos. Cabe mencionar que parte de esta área ya fue incendiada en el año 2012 (Mexico 2012). Esta zona potencial de incendios predicha por el modelo espacial presenta pendientes pronunciadas y el tipo de vegetación es bosque de encino y de pino-encino.

Por otro lado, el modelo también predice pequeñas zonas aisladas potenciales de incendios de $28 \mathrm{Ha}$ en promedio y las cuales suman un total de $869 \mathrm{Ha}$, éstas se ubican en los Ejidos Los Chorros de Tala, la Lobera, la Hiedra, Agua Dulce, los Balnearios Las Tinajas y La Primavera. En estas zonas las variables más importantes son el uso de suelo y el tipo de vegetación.

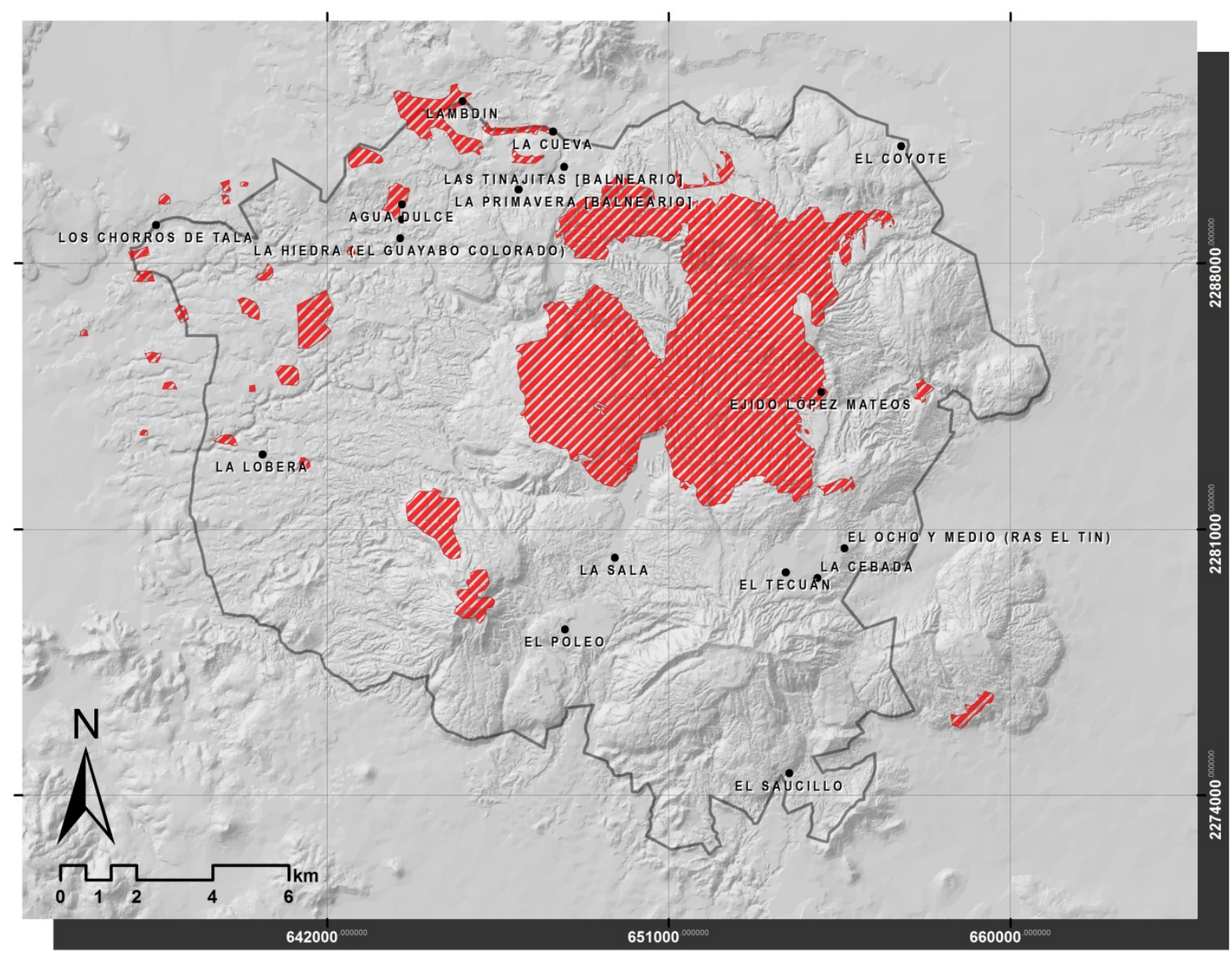

Figura 3. Zonas potenciales de incendio predichas por el modelo espacial.

Este estudio es de las pocas aplicaciones de modelado espacial para determinar zonas potenciales de incendio (Mandallaz y Ye, 1997; García et al., 1999; Muñoz et al., 2005; Pompa, 2012; Pérez-Verdín, 2013), y es el único hasta el momento que en el modelo integra variables de combustibles, meteorológicas, de paisaje y antropológicas y/o de causalidad, bajo un contexto geoespacial.

En este trabajo fue posible integrar las variables sugeridas en el modelo conceptual generado por Pompa (2012), para obtener un modelado espacial y un escenario de predicción, asimismo fue posible determinar las variables más importantes en la generación de incendios en cada sitio de muestreo. 
En estudios anteriores, (Mandallaz et al. 1997; García et al., 1999; Muñoz et al. 2005; Hernández-Leal et al., 2006), los predictores de incendios han sido en general a una escala regional, lo que hace difícil generar conclusiones a escala local. En este estudio, fue necesario generar microambientes dentro del APFFLP para representar la heterogeneidad real del sistema, tanto en los elementos socioeconómicos como en los factores ambientales, y las coberturas de las variables se realizaron a una alta resolución $\left(1 \mathrm{~m}^{2}\right)$ para utilizarlas como predictores, sobre las cuales se desarrolló el modelo de incendios. En este sentido, este estudio representa un esfuerzo novedoso para predecir la probabilidad de incendios a una escala espacial fina.

La heterogeneidad ambiental y complejidad espacial del APFFLP son altas, y están relacionadas con las variables meteorológicas y de paisaje, afectadas por los diferentes niveles de perturbación, usos de suelo, tipo de vegetación así como el tipo de suelo. Esta variabilidad ambiental en un área relativamente pequeña, se refleja en las ocurrencias históricas de los incendios. Por lo que las predicciones de incendios hechas en este trabajo indican a una zona local amplia y aislada, así como a un patrón de pequeñas zonas aisladas.

Los resultados sugieren que la pendiente (variable de paisaje), la humedad relativa (variable meteorológica), el tipo de suelo (variable antropogénica) y el uso de suelo (variable antropogénica); influyen de manera directa en la predicción de incendios en el APFFLP, lo que concuerda parcialmente con lo encontrado por Ruiz y Blanco (2004), quienes apuntan que el riesgo de incendios se incrementa proporcionalmente con la pendiente. El tipo y uso de suelo son variables importantes dentro de la predicción de incendios; ya que como se ha comentado en varias ocasiones, el hombre juega un papel importante en la ocurrencia de los incendios. De acuerdo con Olayo-González ${ }^{1}$, alrededor de $80 \%$ de la superficie afectada por estos eventos ha sido descuidos humanos.

En el caso particular del APFFLP se presentan actividades de agricultura, para lo cual se llevan a cabo incendios controlados durante la zafra en el cultivo de caña de azúcar, y actividades de recreación principalmente en dos áreas. Una de ellas se encuentra del lado norte por la entrada al ejido La Primavera, donde existen balnearios; esta zona es la de mayor carga de visitantes, registrándose cerca de 25,000 visitantes en temporada de Semana Santa. Por el lado oriente, se tiene una segunda vía de acceso en la prolongación de la Avenida Mariano Otero donde no obstante no haber ningún tipo de desarrollo, es la segunda área con mayor afluencia de paseantes, que durante Semana Santa suman 15,000 (Mexico, 2011).

Es posible que el origen potencial de los incendios en el APFFLP esté relacionado con las actividades antropogénicas: el uso de suelo, principalmente en el cultivo de caña de azúcar y las actividades de recreación. Sin embargo la magnitud y extensión de los incendios, así como la dirección de su alcance; podría estar dada por una combinación de variables meteorológicas y de paisaje; principalmente la humedad relativa y la pendiente respectivamente, que por un lado impulsarán el proceso del incendio y por otro lado dificultan el acceso a los brigadistas (Velasco-Herrera et al., 2013; Pompa García y Sensibaugh, 2014).

Estos resultados coinciden con el llamado Triángulo del Fuego, que establece que para que se produzca un incendio se necesitan tres elementos: calor, oxígeno y combustible; basta la ausencia de un elemento para que no se presente un incendio y la Gran Tríada la cual considera el factor topográfico, el tiempo atmosférico y los combustibles como los factores que intervienen en determinar el comportamiento del fuego (Mexico, 2011), De estos factores, el más importante es el tiempo atmosférico, que es el factor más variable y en ocasiones impredecible, lo que exige tener el mayor conocimiento y control para actuar con seguridad y eficiencia. Los elementos del tiempo atmosférico son: Temperatura, Humedad relativa y viento (velocidad y dirección), la interrelación de estos elementos dan como resultado

\footnotetext{
${ }^{1}$ Olayo-González, M. Gerente estatal Conafor Durango. Comunicación Personal. 6 de noviembre. 2012.
} 
condiciones particulares que cambian de manera temporal (a lo largo del día y de las estaciones) y espacial (cañones, laderas, valles), lo que también se refleja en el comportamiento del fuego, en su velocidad de propagación, en la altura de las llamas, en la intensidad de quema entre otros (CENAPRED, 2008).

La predicción del modelo es congruente con los obtenidos en diversos estudios, particularmente en lo referido a las causales de la ocurrencia del fuego (Ávila-Flores et al., 2010; Muñoz et al., 2005; Rodríguez et al., 2008; Meza y Sepúlveda, 2009; Pompa García y Sensibaugh, 2014). No obstante, ninguno incluye una plataforma que integre las variables en un modelo global de índole espacial.

Los hallazgos aquí documentados son muy importantes, sin embargo se requiere una mayor investigación, sobre todo en el diagnóstico del tiempo atmosférico, para lo cual se requiere de un registro sistemático a largo plazo sobre los elementos del clima, para así poder realizar a partir de datos reales, una simulación de diferentes escenarios probables, en función del peso específico de cada variable. Las aplicaciones del modelo pueden ser trascendentales en la dinámica del fuego sobre el ecosistema del APFFLP, en un contexto de cambio climático, y representa una información valiosa para los manejadores del bosque, quienes a partir de este modelo podrían definir sitios con alto potencial de ocurrir incendios y con ello optimizar los planes de prevención y supervisión y estrategias más efectivas de combate.

El modelo espacial generado presenta una solidez conceptual para determinar zonas potenciales de incendios, mediante su integración a partir de componentes de paisaje, meteorológicos, antropogénicos y/o causales y de combustibles. Su implementación a través de la plataforma desarrollada, constituye un aporte científico relevante y vanguardista en el modelaje de zonas potenciales de incendios en el APFFLP.

\section{CONCLUSIONES}

El modelo espacial desarrollado demostró ser una metodología confiable para predecir el potencial de ocurrencia de incendios en el APFFLP. En él, se destacan zonas potenciales, asiladas y pequeñas al oriente del polígono del APFFLP, las cuales históricamente se han quemado debido a las actividades propias para el cultivo de la caña de azúcar. Se destaca también una zona más grande ubicada en la parte noroeste del polígono que probablemente se incendie por las actividades de recreación y se magnifique por las condiciones topográficas y meteorológicas presentadas en esos sitios. La heterogeneidad ambiental del APFFLP, las actividades antropogénicas, las variables de uso de suelo, y la pendiente influyen en la ocurrencia del fuego.

Es esperanza para los autores, que este estudio pueda ayudar a identificar los sitios específicos en los que se recomienda realizar acciones preventivas específicas contra los incendios por parte de las autoridades correspondientes, y que tales esfuerzos puedan orientarse en una estrecha coordinación entre las comunidades locales, autoridades y la academia. De las variables que resultaron con alta importancia en la ocurrencia del fuego, la única que puede ser manipulada y controlada por el hombre es el uso de suelo, de tal forma que es en esta variable donde se debe incidir a fin de evitar la presencia de incendios forestales de la misma magnitud como las presentadas en los años 1998, 2005 y 2012.

\section{AGRADECIMIENTOS}

Agradecemos al Biol. Gerardo Cabrera Orozco, por su ayuda y cooperación en campo, así como a los miembros de SAP, Servicios Ambientales Profesionales. También agradecemos a la Biol. Karla Gutiérrez García por su apoyo en la construcción del SIG, y en 
la edición de los mapas de la APFFLP. Al CONACYT por su apoyo con la beca posdoctoral del Dr. José Luis Ibarra-Montoya.

\section{REFERENCIAS}

ALMEIDA, R. Forest fire risk areas and definition of the prevention priority planning actions using GIS. In: EUROPEAN CONFERENCE ON GEOGRAPHICAL INFORMATION SYSTEMS - EGIS, 5., 1994, Paris. Proceedings... Paris: EGIS Fundation, 1994.

ÁVILA-FLORES, D.; POMPA-GARCÍA, E. Spatial analysis of fire occurrence in the Durango State. Revista Chapingo. Serie Ciencias Forestales, v. 16, n. 2, p. 253-260, 2010 .

ÁVILA-FLORES, D.; POMPA-GARCÍA, M.; ANTONIO-NEMIGA, S.; RODRIGUEZTREJO, D.; VARGAS-PÉREZ, E.; SANTILLÁN-PEREZ, J. Driving factors for forest fire occurrence in Durango State of Mexico: A geospatial perspective. Chinese Geographical Science, v. 20, n. 6, p. 491-497, 2010. http://dx.doi.org/10.1007/s11769010-0437-x

CENTRO NACIONAL DE PREVENCIÓN DE DESASTRES - CENAPRED. Incendios Forestales. Zapopan: CONAFOR, 2008. P. 44.

CHUVIECO, E.; SALAS, F.; VEGA, C. Remote sensing and GIS for long-term fire risk mapping. Mega fires Project. In: CHUVIECO, E. (ed), A review of remote sensing methods for the study of large wildland fires. Madrid: Universidad de Alcalá, 2000. p. 91-107.

COMISIÓN NACIONAL PARA EL CONOCIMIENTO Y USO DE LA BIODIVERSIDAD - CONABIO. Incendio Forestales. 2014. Disponible en: http://www.conabio. gob.mx/informacion/geo_espanol/doctos/imagsatelite.html. Acceso en: 20 mayo 2014.

COMISIÓN NACIONAL FORESTAL - CONAFOR. Reporte nacional de incendios forestales. Guadalajara, Disponible en: http://www.mexicoforestal.gob.mx/files/ 120427reportenacionalincendis. Acceso en: 15 mayo 2014.

COMMONWEALTH SCIENTIFIC AND INDUSTRIAL RESEARCH ORGANIZATION CSIRO Forestry and Forest Products. Bushfire Behavior and Management. 2000. Disponible en: http://www.ffp.csiro.au/nfm/fbm/. Acceso en: 15 mayo 2014.

DEEMING, J.; BURGAN, R.; COHEN, J. D. The National Fire Danger Rating System. Washington, D.C.: USDA Forest Service, 1978.

DELANEY, J. Geographical information systems an introduction. Oxford: University press, 1999. $194 \mathrm{p}$.

DRYRY, S.; VEBLEN, T. Spatial and temporal variability in fire occurrence within the Las Bayas forestry reserve, Durango, México. Plant Ecology, v. 197, p. 299-316, 2008. http://dx.doi.org/10.1007/s11258-007-9379-5

FLASSE, P.; CECCATO, P. A contextual algorithm for AVHRR fire detection. International Journal of Remote Sensing, v. 17, p. 419-424, 1996. http://dx.doi.org/10.1080/01431169608949018

GARCÍA, E. Modificaciones al sistema de clasificación climática de Koppen. México: Instituto de Geografía, UNAM, 1973. 256 p. 
GARCÍA, V.; WOODARD, S.; ADAMOWICZ, P.; LEE, B. Dos modelos para la predicción de incendios forestales en Whitecourt Forest, Canada. Investigación Agraria, Sistemas y Recursos Forestales, v. 8, n. 1, p. 5-23, 1999.

GIGLIO, L.; DESCLOITRES, J.; JUSTICE, O.; KAUFMAN, J. An enhanced contextual fire detection algorithm for MODIS. Remote Sensing of Environment, v. 87, p. 273-282, 2003. http://dx.doi.org/10.1016/S0034-4257(03)00184-6

GOLLBERG, E.; NEUENSCHWANDER, F.; RYAN, C. Introduction: Integrating spatial technologies and ecological principles for a new age in fire management. International Journal of Wild land Fire, v. 10, p. 263-265, 2001.

http://dx.doi.org/10.1071/WF01047

GOUMA, V.; CHRONOPOULOU-SERELI, A. Wild land Fire danger zoning-A methodology. International Journal of Wildland Fire, v. 8, n. 1, p. 37-43, 1998. http://dx.doi.org/10.1071/WF9980037

GUTIERREZ, M.; MONEDERO, G.; SANDIA, A. Diseño de un mapa de incendios de vegetación en el Parque Nacional El Ávila apoyado en Modelos Digitales del Terreno. In: SIMPOSIO LATINOAMERICANO DE PERCEPCIÓN REMOTA 7., 1997, Mérida. Memorias..., Caracas: SELPER/Unidade Técnica de Sistemas. Instituto de Ingeneria, 1997.

HERNÁNDEZ-LEAL, P. A.; ARBELO, M.; GONZÁLEZ-CALVO, A. Fire risk assessment using satellite data. Advances in Space Research, v. 37, p. 741-746, 2006. http://dx.doi.org/10.1016/j.asr.2004.12.053

HUERTA-MARTÍNEZ, F. M.; IBARRA-MONTOYA, J. L. 15 Años de incendios en el Bosque La Primavera (Jalisco, México): Un acercamiento a sus posibles causas y consecuencias. CienciaUAT, v. 8, n. 2, p. 32-43, 2014.

INSTITUTO NACIONAL DE ESTADÍSTICA Y GEOGRAFIA - INEGI. Continuo Nacional del conjunto de datos vectoriales de uso de suelo y vegetación, 1:1 250 000 serie II. Jalisco, 2014a.

INSTITUTO NACIONAL DE ESTADÍSTICA Y GEOGRAFIA - INEGI. Continuo Nacional del conjunto de datos geográficos de la carta topográfica, 1:1 250000 serie II. Jalisco, 2014b.

LEATHWICK, J.; BRIGGS, C. M. Spatial prediction of wildfire hazard across. New Zealand: New Zealand Fire Service Commission, 2001.

LEE, B.; ALEXANDER, M.; HAWKES, B.; LYNHAM, T.; STOCK, B.; ENGLEFIELD, P. Information system in support of wild land fire management decision making in Canada. Computers and Electronics in Agriculture, v. 37, n. 1-3, p. 185-198, 2002. http://dx.doi.org/10.1016/S0168-1699(02)00120-5

MAGAÑA, O. Índices de peligro de incendios forestales. México: Instituto Nacional de Investigaciones Forestales, 1985. 
MANDALLAZ, D.; YE, R. Prediction of forest fires with Poisson models. Canadian Journal of Forest Research, v. 27, n. 10, p. 1685-1694, 1997. http://dx.doi.org/10.1139/x97-103MANOS, P.; DOYLE, J.; NIXON, K. Phylogeny, Biogeography, and Processes of Molecular Differentiation in Quercus Subgenus Quercus (Fagaceae). Molecular Phylogenetics and Evolution, v. 12, n. 3, p. 333-349, 1999. http://dx.doi.org/10.1006/mpev.1999.0614

MARCOZZI, M.; BOVIO, G.; MANDALLAZ, D.; BACHMANN, P. Influence of the weather on the forest fire danger index in Ticino Canton. Schweizerische Zeitschrift fur Forstwesen, v. 145, n. 3, p. 183-199, 1994.

MATHUR, R.; GOGATE, M.; MITTAL, R. Forest fire danger rating índices base don climate-a case study of West Dehra Dun Forest Division. Indian Forester, v. 110, n. 3, p. 223-241, 1984.

MEXICO. Secretaria de Medio Ambiente y Recursos Naturales - SEMARNAT. Programa de Manejo. Área de Protección de Flora y Fauna La Primavera. México, 2000. p.131.

MEXICO. Secretaria de Medio Ambiente y Recursos Naturales - SEMARNAT. Programas y Acciones en Reforestación, Conservación y Restauración de Suelos, Incendios Forestales y Sanidad Forestal de Ecosistemas forestales. Programa Nacional contra Incendios Forestales. México, 2011. p 65-106.

MEXICO. Secretaria de Medio Ambiente y Recursos Naturales - SEMARNAT. Programas y Acciones en Reforestación, Conservación y Restauración de Suelos, Incendios Forestales y Sanidad Forestal de Ecosistemas forestales. Programa Nacional contra Incendios Forestales. Mexico, 2012. p. 60-97.

MEZA, R.; SEPÚLVEDA, J. Caracterización del terreno y su influencia en los incendios forestales en Baja California, México. La Paz: INIFAP, 2009. www.mappinginteractivo.com/plantilla-egeo.asp?idarticulo=1603. Acceso en: 17 febrero de 2012.

MUÑOZ, R.; TREVIÑO, E.; VERÁSTEGUI, J.; JIMÉNEZ, J.; AGUIRRE, O. Desarrollo de un modelo espacial para la evaluación del peligro de incendios forestales en la Sierra Madre Oriental de México. Investigaciones Geográficas, v. 56, p. 101-117, 2005.

NIXON, K.; MULLER, C. The Quercus hypoxantha complex (Fagaceae) in Northeastern Mexico. Brittonia, v. 45, p.146-153, 1993. http://dx.do.org/10.2307/2807497

PAROLO, G.; ROSSI, G.; FERRARINI, A. Toward improved species niche modelling: Arnica montana in the Alps as a case study. Journal of Applied Ecology, n. 45, p. 1410-1418, 2008. http://d.doi.org/10.1111/j.1365-2664.2008.01516.x

PÉREZ-VERDÍN, G.; MÁRQUEZ-LINARES, M.; CORTÉS-ORTÍZ, A.; SALMERÓNMACÍAS, M. Análisis espacio-temporal de la ocurrencia de incendios forestales en Durango, México. Madera y Bosques, v. 19, n. 2, p. 37-58, 2013.

PHILLIPS, S. J.; ANDERSON, R. P.; SCHAPIRE, R. E. Maximum entropy modeling of species geographic distributions. Ecological Modeling, n. 190, p. 231-259, 2006. http://dx.doi.org/10.1016/j.ecolmodel.2005.03.026

PHILLIPS, S. J.; DUDIK, M. Modeling of species distributions with Maxent: new extensions and a comprehensive evaluation. Ecography, v. 31, p. 161-175, 2008. http://dx.doi.org/10.1111/j.0906-7590.2008.5203.x 
POMPA, G. Sistema de información geográfica de los ecosistemas de Durango. Mapa de climas. 2012. http://www.ujed.mx/sigeed/inicio.aspx. Acceso en: 01 febrero de 2012.

POMPA-GARCÍA, M.; SENSIBAUGH, M. Ocurrencia de incendios forestales y su teleconexión con fenómenos ENSO. CienciaUAT, v. 27, n. 2, p. 06-10, 2014.

RODRIGUEZ, T.; RAMÍREZ, H.; TCHIKOUE, H.; SANTILLÁN, J. Factores que inciden en la siniestralidad de los incendios forestales. Ciencia Forestal en México, v. 33, n. 104, p. 38-57, 2008.

RUIZ L., V.; BLANCO J., L. Comportamiento del fuego y evaluación del riesgo por incendios en las áreas forestales de México: un estudio en el Volcán la Malinche. In: Incendios forestales en Mexico. Metodos De Evaluación. Mexico: Universidad Nacional Autónoma de Mexico, 2004.

SÁNCHEZ, J. Los incendios forestales y las prioridades de investigación en México. México: Congreso Forestal Mexicano, 1989. p. 719-723.

SANTILLAN, P. Sistema para determinar indicadores de peligro de incendio forestal. Acuerdo de cooperación en materia forestal entre México y Finlandia. Helsinque: [s.n.], 1993. (Informe Técnico, n. 14)

SEPÚlVEDA, B.; ZUÑIGA, C.; OLGUÍN, E.; GOMERO, P. Implementación de un sistema de información geográfica para la prevención de incendios forestales en Baja California. Mexico: INIFAP; SAGAR, 1999.

SEPÚlVEDA, R.; MEZA, W.; ZUÑIGA, G.; SOLÍS, G.; OLGUÍN, E. SIG para determinar riesgo de incendios forestales en el noroeste de México. Mexico: Instituto Nacional de Investigaciones Forestales, Agrícolas y Pecuarias, 2001. p. 37.

SOKAL, R.; ROHLF, J. Biometry. The principles and practice of statistics in biological research. New York: State University of New York, 1995. 887 p.

STOLYARCHUK, L. Structure of the forest fire seasons in the regions west and east of lake Baikal. Lesnoe-Khozyaistvo, n. 7, p. 57-58, 1979.

VÁZQUEZ, M.; VALENCIA, S.; NIXON, K. Notes on red oaks (Quercus sect. Lobatae) in eastern Mexico, with description of a new species. Quercus hirtiifolia. Brittonia, v. 56, p. 136-142, 2004. http://dx.doi.org/10.1663/0007-196X(2004)056[0136:NOROQS] 2.0.CO;2

VELASCO-HERRERA, J.; FLORES-GARNICA, J.; MARQUEZ-AZÚA, B.; LÓPEZ, S. Áreas de respuesta homogénea para el muestreo de combustibles forestales. Revista Mexicana de Ciencias Forestales, v. 4, n. 15, p. 41-54, 2013.

WYBO, J.; GUARNIERI, F.; RICHARD, B. Forest-fire danger assessment methods and decision support. Safety Science, v. 20, n. 1, p. 61-70, 1995. http://dx.doi.org/10.1016/0925-7535(94)00067-D

ZAPATA, P. Determinación de indicadores de peligro potencial de incendios forestales con base en la cuantificación del material combustible. Chapingo: UACH, 1991. 